\title{
Augmented Burnett-Equation Solutions over Axisymmetric Blunt Bodies in Hypersonic Flow
}

\author{
Xiaolin Zhong* and Gregory H. Furumoto ${ }^{\dagger}$ \\ University of California, Los Angeles, Los Angeles, California 90095
}

\begin{abstract}
We obtained augmented Burnett-equation solutions and conducted some preliminary studies on the extent to which the Burnett equations can be used in estimating the departure from the NavierStokes model for hypersonic flow over axisymmetricblunt bodies. The augmented Burnett equation solutions were restricted to the flow inside the bow shock in the stagnation region because of the uncertainty of boundary conditions. On one hand, the present results partly confirm the past observation that the Burnett equations can only make insignificant improvement over the Navier-Stokes equations when the latter are adequate, but the Burnett equations fail when the NavierStokes equations become inadequate. On the other hand, though both the NavierStokes and Burnett equations are inaccurate for flow inside a strong shock, to some extent the augmented Burnett solutions do seem to agree better with results obtained by the direct simulation Monte Carlo technique than the Navier-Stokes solutions do. Further studies are needed on the validity and significance of the augmented Burnett solutions for such flowfields.
\end{abstract}

\begin{tabular}{|c|c|}
\hline & Nomenclature \\
\hline$c_{\infty}$ & $\begin{array}{l}=\text { freestream most probable molecular } \\
\text { speed, } \mathrm{m} / \mathrm{s}\end{array}$ \\
\hline$c_{v}$ & $\begin{aligned} &= \text { specific heat at constant volume, } \\
& \mathrm{J} / \mathrm{K} \mathrm{kg}\end{aligned}$ \\
\hline$e$ & $=$ total energy per unit volume, $\mathrm{J} / \mathrm{m}^{3}$ \\
\hline $\boldsymbol{F}, \boldsymbol{G}, \boldsymbol{F}^{\prime}$ & $=$ flux vectors \\
\hline$h_{i}$ & $\begin{aligned}= & \text { metric coefficients in cylindrical } \\
& \text { coordinates }\end{aligned}$ \\
\hline$K n_{\infty}$ & $=$ freestream Knudsen number, $\lambda_{\infty} / \bar{r}$ \\
\hline$M_{\infty}$ & $=$ freestream Mach number \\
\hline$n$ & $=$ normal unit vector in $(x, y)$ plane \\
\hline $\operatorname{Pr}$ & $=$ Prandtl number \\
\hline$p$ & $=$ pressure, $\mathrm{N} / \mathrm{m}^{2}$ \\
\hline $\boldsymbol{q}, \boldsymbol{q}^{(B)}, \boldsymbol{q}^{(1)}, \boldsymbol{q}^{(2)}, \boldsymbol{q}^{(a)}$ & $=$ heat-flux vectors, $\mathrm{J} / \mathrm{m}^{2} \mathrm{~s}$ \\
\hline$q_{x}, q_{y}$ & $\begin{array}{l}=\text { heat-flux components } \mathrm{J} / \mathrm{m}^{2} \mathrm{~s} \\
=\text { gas constant, } \mathrm{J} / \mathrm{kg} \mathrm{K}\end{array}$ \\
\hline$R e_{\infty}$ & $=$ Reynolds number \\
\hline $\bar{r}$ & $=$ radius of sphere, $\mathrm{m}$ \\
\hline$r, \epsilon, z$ & $=$ cylindrical coordinates \\
\hline$S_{i+\frac{1}{2}}$ & $=$ cell interface area, $\mathrm{m}^{2}$ \\
\hline$S_{i j}^{i+\overline{2}}$ & $\begin{aligned}= & \text { computational cell surface area in the } \\
& (x, y) \text { plane, } \mathrm{m}^{2}\end{aligned}$ \\
\hline$T$ & $=$ temperature, $\mathbf{K}$ \\
\hline & $=$ reference temperature, $300 \mathrm{~K}$ \\
\hline$T_{j}, T_{j k}$ & $\begin{aligned}= & \text { temperature derivatives in cylindrical } \\
& \text { coordinates }\end{aligned}$ \\
\hline$T_{\text {wall }}$ & $=$ wall temperature, $\mathbf{K}$ \\
\hline$t$ & $=$ time, $\mathbf{s}$ \\
\hline $\boldsymbol{u}, u, v$ & $=$ velocity vector and components, $\mathrm{m} / \mathrm{s}$ \\
\hline$u_{i}$ & $\begin{aligned}= & \text { velocity components in cylindrical } \\
& \text { coordinates, } \mathrm{m} / \mathrm{s}\end{aligned}$ \\
\hline$u_{i j}, u_{i j k}$ & $=$ velocity derivatives \\
\hline$U, \tilde{U}$ & $\begin{array}{l}=\text { flow-variable vector and its } \\
\text { cell-average values }\end{array}$ \\
\hline$V_{i j}$ & $=$ Computational cell volume, $\mathrm{m}^{3}$ \\
\hline$x_{i}$ & $\begin{aligned}= & \text { coordinates in cylindrical } \\
& \text { coordinates, } \mathrm{m}\end{aligned}$ \\
\hline
\end{tabular}

Presented as Paper 94-1959 at the AIAA/ASME 6th Joint Thermophysics and Heat TransferConference, Colorado Springs, CO, June 20-23,1994; received July 26,1994; revision received Jan. 8,1995; accepted for publication Jan. 17, 1995. Copyright (C) 1995 by the American Institute of Aeronautics and Astronautics, Inc. All rights reserved.

*Assistant Professor, Mechanical, Aerospace and Nuclear Engineering Department. Member AIAA.

${ }^{\dagger}$ Graduate Student, Mechanical, Aerospace and Nuclear Engineering Department.

$$
\begin{aligned}
& x, y, z=\text { Cartesian coordinates } \\
& \text { a }=\text { thermal accommodation coefficient } \\
& \gamma \quad=\text { ratio of specific heats } \\
& \delta \quad=\text { unit tensor } \\
& \kappa=\quad=\text { coefficient of thermal conductivity, } \\
& \mathrm{J} / \mathrm{m} \mathrm{s} \mathrm{K} \\
& \lambda=\text { mean free path, } 16 \mu /(5 \rho \sqrt{2 \pi R T}) \\
& \mu \quad=\text { viscosity coefficient, } \mathbf{s N} / \mathrm{m}^{2} \\
& \mu_{0} \quad=\text { reference viscosity coefficient, } \\
& 2.2695 \times 10^{-5} \mathrm{~kg} / \mathrm{m} \mathrm{s} \\
& P \quad=\text { density, } \mathrm{kg} / \mathrm{m}^{3} \\
& \rho_{j}, \rho_{j k} \quad=\text { density derivatives in cylindrical } \\
& \text { coordinates } \\
& \text { a }=\text { momentum reflection coefficient on } \\
& \boldsymbol{\sigma}, \boldsymbol{\sigma}^{(B)}, \boldsymbol{\sigma}^{(1)}, \boldsymbol{\sigma}^{(2)}, \boldsymbol{\sigma}^{(a)} \quad \begin{array}{l}
\text { the wall } \\
=
\end{array} \\
& \sigma_{x}, \sigma_{x y}, \sigma_{x}, \sigma_{y} \quad=\text { components of stress tensors, } \mathrm{N} / \mathrm{m}^{2} \\
& \omega_{i}, \theta_{i}, \alpha_{i}, \beta_{i}, \gamma_{i} \quad=\text { constants in the Burnett stress and } \\
& \text { heat-flux terms }
\end{aligned}
$$

\section{Introduction}

$\mathbf{R}^{\mathrm{s}}$ ECENTLY, there have been many studies of the Burnett equations to investigate if they can be used as a computational fluid dynamics (CFD) model to study slightly rarefied hypersonic flows. ${ }^{1-8}$ However, the usefulness of the Burnett equations is still uncertain. The purpose of this paper is to obtain augmented Burnett solutions and to conduct some preliminary studies on the extent to which the augmented Burnett equations can be used in estimating the departure from the Navier-Stokes model in an axisymmetric hypersonic flowfield. In order to obtain unique augmented Burnett solutions, we only consider hypersonic flow past a sphere near the stagnation region.

The Bumett equations were first studied in 1950s. It was observed $^{y}$ that the Burnett equations can only make insignificant improvement over the Navier-Stokes equations when the latter equations are adequate, but the Burnett equations fail when the Navier-Stokes equations become inadequate. There are many unanswered questions and unsolved issues concerning the Bumett equations. Two of those questions and issues are 1) the well-posedness of these equations is questionable ${ }^{10}$ and 2 ) no satisfactory boundary conditions are available for these higher-order equations. Thus, unique multidimensional Burnett solutions in general are difficult to obtain.

Numerical solutions of the conventional Burnett equations for one-dimensional shock-wave structure have been difficult to 
obtain because the equations are unstable to small-wavelength disturbances." As a result, if the Burnett equations are solved as an initial boundary-value problem, the numerical computations will be unstable when fine computational grids are used. Therefore, an augmented version of the Burnett equations containing higher-order terms was proposed ${ }^{4}$ to stablize the computations. Though these ad hoc stabilizing terms are not unique, the solutions of the augmented Burnett equations for one-dimensional shock structure compare well with direct simulation Monte Carlo (DSMC) results. The conventional and augmented Burnett solutions have been found to be superior to the Navier-Stokes ones for one-dimensional shock-wave structure. ${ }^{1,3,4,12}$

For multidimensional flowfields containing solid boundaries, the Burnett solutions are more difficult to obtain because these higherorder equations require additional boundary conditions. The effect of the boundary conditions on the uniqueness of Burnett solutions for Couette flow was demonstrated in Ref. 13. Though no satisfactory boundary conditions for the Burnett equations ${ }^{8,13}$ are currently available, unique solutions of the augmented Bumett equations can still be obtained for some special cases of multidimensional flows if the Burnett terms are negligible in the boundary layer on the wall. An example of such flowfields is hypersonic flow past a blunt body near the stagnation point at a small $\boldsymbol{K} \boldsymbol{n}$,, moderate $M_{\infty}$, and coldwall boundary condition. In this flowfield, the flow density increases significantly across the bow shock, and nonequilibrium mainly occurs inside the bow shock, which is away from the wall. This argument can be checked by examining the distribution of $\left|\sigma_{i j} / p\right|$ across the stagnation line. Therefore, unique solutions to the augmented Burnett equations can be obtained, because these equations are different from the Navier-Stokesequations on the part of the flowfield mainly inside the shock layer, and the Burnett equations reduce to the Navier-Stokes equations on the wall. For this reason, augmented Bumett solutions for planar two-dimensional hypersonic flow past a cylinder near the stagnation point have been obtained. ${ }^{4,6,7}$ The results show that two-dimensional augmented Bumett solutions for such flow agree reasonably well with the DSMC results. On the other hand, Burnett solutions for axisymmetric hypersonic flow, which is closer to practical hypersonic flow around blunt bodies than planar two-dimensional flow, have not been available.

In this paper, we first derived three-dimensional components of the augmented Burnett equations from their tensor forms. Then, we obtained the augmented Burnett numerical solutions for the axisymmetric hypersonic flow past a sphere. Finally, we examined the differences among the numerical solutions to the augmented Burnett equations, the Navier-Stokesequations, Navier-Stokes-basedequations, and a DSMC solutions for a test case. With regard to the past observation' stated in the beginning of this section on the usefulness of the Bumett equations, the current results seem to agree with the observation except in the temperature profiles. The augmented Burnett solutions are different from the Navier-Stokes equations mainly in the temperature distributions. Though both the NavierStokes and Burnett equations are inaccurate inside the strong bow shock, the augmented Burnett solutions do seem to agree, to some extent, better with DSMC results than with the Navier-Stokesequations. This property of the augment Burnett equations may be significant for studying hypersonic flow where accurate prediction of the temperature profiles is important, if the augmented Burnett equations are indeed proven to be more adequate.

\section{Burnett Equations in Tensor Form}

\section{Burnett Equations}

The governing equations for the continuum description of fluid flow are the following conservation equations of mass, momentum, and energy:

$$
\begin{gathered}
\frac{\partial \rho}{\text { at }}+\boldsymbol{v} \cdot[p u]=0 \\
\frac{a p u}{\mathrm{at}}+\nabla \cdot[\rho u \boldsymbol{u}+p \delta+\sigma]=0 \\
\frac{\partial e}{\mathrm{at}}+\nabla \cdot[(e+p) \boldsymbol{u}+\boldsymbol{u} \cdot \boldsymbol{\sigma}+q]=0
\end{gathered}
$$

where

$$
\begin{gathered}
e_{i}=c_{v} T \\
p=\rho R T \\
e=\rho\left(e_{i}+\frac{1}{2} u \cdot u\right)
\end{gathered}
$$

The Burnett equations use second-orderconstitutive relations in the governing equations for $\mathbf{a}$ and $\boldsymbol{q}$ derived by the Chapman-Enskog method $^{14,15}$ :

$$
\begin{aligned}
\boldsymbol{\sigma}^{(B)} & =\boldsymbol{\sigma}^{(1)}+\boldsymbol{\sigma}^{(2)} \\
& =-2 \mu \overline{\nabla \boldsymbol{u}}+\frac{\mu^{2}}{p}\left\{\omega_{1}(\nabla \cdot \boldsymbol{u}) \overline{\nabla \boldsymbol{u}}+\omega_{2}\left[-\overline{\nabla\left(\frac{1}{\rho} \nabla p\right)}\right.\right. \\
& -\overline{\nabla \boldsymbol{u} \cdot \nabla \boldsymbol{u}}-2 \nabla \boldsymbol{u} \cdot \nabla \boldsymbol{u}+\omega_{3} R \overline{\nabla \nabla T}+\omega_{4} \frac{1}{\rho T} \overline{\nabla p \nabla T} \\
& \left.+\omega_{5} \frac{R}{\boldsymbol{T}} \overline{\nabla T \nabla \bar{T}}+\omega_{6} \overline{\overline{\nabla \boldsymbol{u}} \cdot \overline{\bar{\nabla} \boldsymbol{V}}}\right\}
\end{aligned}
$$

and

$$
\begin{aligned}
& \boldsymbol{q}^{(B)}=\boldsymbol{q}^{(1)}+\boldsymbol{q}^{(2)}=-\kappa \nabla T+\frac{\mu^{2}}{\rho}\left\{\theta_{1} \frac{1}{T} \nabla \cdot \mathbf{u} \nabla T\right. \\
& +\theta_{2} \frac{1}{T}\left[\frac{2}{3} \nabla(T \nabla \cdot u)+2 \nabla \boldsymbol{u} \cdot \nabla T\right]+\theta_{3} \stackrel{1}{\stackrel{f}{p}} \nabla p \cdot \bar{\nabla} \boldsymbol{u} \\
& \left.+\theta_{4} \nabla \cdot \bar{\nabla} u+85 \bar{f} \nabla T \cdot \bar{\nabla} U\right\}
\end{aligned}
$$

where $\boldsymbol{\sigma}^{(1)}$ and $\boldsymbol{q}^{(1)}$ are the first-order constitutive relations in the Navier-Stokes equations, and $\boldsymbol{\sigma}^{(2)}$ and $\boldsymbol{q}^{(2)}$ are the second-order corrections introduced in the Burnett equations. A bar over a tensor designates a nondivergent symmetrical tensor. The coefficients $\omega_{i}$ and $\theta_{i}$ are constants depending on the molecular repulsive force model used. For the hard-sphere gas model, $\omega_{1}=4.056, \omega_{2}=$ 2.028, $\omega_{3}=2.418, \omega_{4}=0.681, \omega_{5}=0.219, \omega_{6}=7.424, \theta_{1}=$ $11.644, \theta_{2}=-5.822, \theta_{3}=-3.090, \theta_{4}=2.418$, and $\theta_{5}=25.157$.

Using a linearized stability analysis, Bobylev' ${ }^{1}$ showed that the conventional Burnett equations are unstable to disturbances of small wavelength. When the equations are solved as an initial boundary-value problem in a time-marching numerical method, the instability of the Burnett equations will make obtaining numerical solutions difficult. Zhong et al. ${ }^{4}$ proposed a set of the "augmented" Bumett equations to stabilize the conventional Burnett equations while maintaining their second-order accuracy. The augmented Bumett equations were formed by augmenting the conventional Bumett stress and heat-flux terms with the following third-order derivative terms:

$$
\begin{gathered}
\boldsymbol{\sigma}=\boldsymbol{\sigma}^{(B)}+\boldsymbol{\sigma}^{(a)} \\
\boldsymbol{q}=\boldsymbol{q}^{(B)}+\boldsymbol{q}^{(a)}
\end{gathered}
$$

where

$$
\begin{gathered}
\sigma^{(a)}=\begin{array}{c}
\left.\left.\mu^{3} \cdot \frac{3}{2} \omega_{7} R T \overline{\nabla\left(\nabla^{2} \boldsymbol{u}\right.}\right)\right\} \\
p^{2} \frac{\mu^{3}}{p \rho}
\end{array}\left\{\theta_{7} R \nabla\left(\nabla^{2} T\right)+\theta_{6} \frac{R T}{\rho} \nabla\left(\nabla^{2} \rho\right)\right\}
\end{gathered}
$$

where $\omega_{7}=2 / 9, \theta_{6}=-5 / 8$ and $\theta_{7}=11 / 16$. The augmented terms, whose forms and coefficients are not unique, are used in order to stabilize the numerical computations of the Burnett equations.

\section{Components of Burnett Terms in Cylindrical Coordinates}

The components of the Burnett stress and heat-flux terms in cylindrical coordinates shown in Fig. 1 are derived from their general tensor forms given by Eqs. (7) and (8) as follows: 


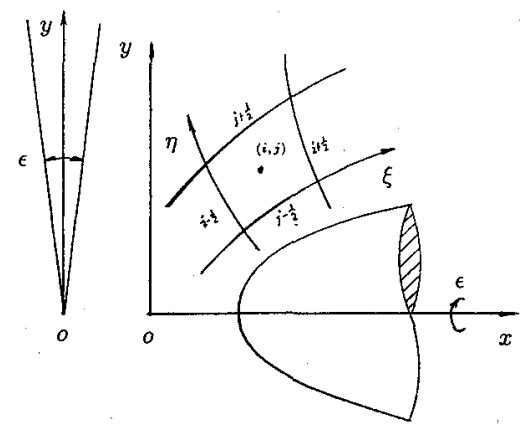

Fig. 1 Cylindrical coordinates for expanding the three-dimensional Burnett equations.

$$
\begin{aligned}
& \sigma_{11}^{(2)}=\frac{\mu^{2}}{p}\left(\alpha_{1} u_{11}^{2}+\alpha_{2} u_{11} u_{22}+\alpha_{3} u_{22}^{2}+\alpha_{4} u_{12} u_{21}\right. \\
& +\alpha_{5} u_{12}^{2}+\alpha_{6} u_{21}^{2}+\alpha_{2} u_{11} u_{33}+\alpha_{18} u_{22} u_{33} \\
& +\alpha_{3} u_{33}^{2}+\alpha_{5} u_{13}^{2}+\alpha_{4} u_{13} u_{31}+\alpha_{6} u_{31}^{2} \\
& +\alpha_{19} u_{23}^{2}+\alpha_{20} u_{23} u_{32}+\alpha_{21} u_{32}^{2}+\alpha_{7} R T_{11} \\
& +\alpha_{8} R T_{22}+\alpha_{9} \frac{R T}{\rho} \rho_{11}+\alpha_{10} \frac{R T}{\rho} \rho_{22}+\alpha_{10} \frac{R T}{\rho} \rho_{33} \\
& +\alpha_{8} R T_{33}+\alpha_{11} \frac{R T}{\rho^{2}} \rho_{1}^{2}+\alpha_{14} \frac{R T}{\rho^{2}} \rho_{2}^{2}+\alpha_{12} \frac{R}{\rho} T_{1} \rho_{1} \\
& +\alpha_{13} \frac{R}{T} T_{1}^{2}+\alpha_{15} \frac{R}{\rho} T_{2} \rho_{2}+\alpha_{16} \frac{R}{T} T_{2}^{2}+\alpha_{14} \frac{R T}{\rho^{2}} \rho_{3}^{2} \\
& \left.+\alpha_{15} \frac{R}{\rho} T_{3} \rho_{3}+\alpha_{16} \frac{R}{T} T_{3}^{2}\right)+\frac{\mu^{2}}{p}\left(\alpha_{2} \frac{u_{1}}{r} u_{11}+\alpha_{22} \frac{u_{1}}{r} u_{22}\right. \\
& +\alpha_{18} \frac{u_{1}}{r} u_{33}+\alpha_{3} \frac{u_{1}^{2}}{r^{2}}+\alpha_{24} \frac{u_{2}}{r} u_{12}+\alpha_{25} \frac{u_{2}}{r} u_{21} \\
& \left.+\alpha_{5} \frac{u_{2}^{2}}{r^{2}}+\alpha_{8} R \frac{T_{1}}{r}+\alpha_{10} \frac{R T}{\rho r} \rho_{1}\right) \\
& \sigma_{22}^{(2)}=\frac{\mu^{2}}{p}(\cdots)+\frac{\mu^{2}}{p}\left(\alpha_{2} \frac{u_{1}}{r} u_{11}+\alpha_{17} \frac{u_{1}}{r} u_{22}\right. \\
& +\alpha_{2} \frac{u_{1}}{r} u_{33}+\alpha_{27} \frac{u_{1}^{2}}{r^{2}}+\alpha_{23} \frac{u_{2}}{r} u_{12}+\alpha_{25} \frac{u_{2}}{r} u_{21} \\
& \left.+\alpha_{6} \frac{u_{2}^{2}}{r^{2}}+\alpha_{7} R \frac{T_{1}}{r}+\alpha_{9} \frac{R T}{\rho r} \rho_{1}\right) \\
& \sigma_{33}^{(2)}=\frac{\mu^{2}}{p}(\cdots)+\frac{\mu^{2}}{p}\left(\alpha_{18} \frac{u_{1}}{r} u_{11}+\alpha_{22} \frac{u_{1}}{r} u_{22}\right. \\
& +\alpha_{2} \frac{u_{1}}{r} u_{33}+\alpha_{3} \frac{u_{1}^{2}}{r^{2}}+\alpha_{26} \frac{u_{2}}{r} u_{12}+\alpha_{28} \frac{u_{2}}{r} u_{21} \\
& \left.+\alpha_{21} \frac{u_{2}^{2}}{r^{2}}+\alpha_{8} R \frac{T_{1}}{r}+\alpha_{10} \frac{R T}{\rho r} \rho_{1}\right)
\end{aligned}
$$

where

$$
\begin{gathered}
x_{1}=r, \quad x_{2}=\varepsilon, \quad x_{3}=z \\
h_{1}=1, \quad h_{2}=r=x_{1}, \quad h_{3}=1 \\
u_{i j}=\frac{\partial u_{i}}{h_{j} \partial x_{j}}, \quad u_{i j k}=\frac{\partial u_{i j}}{h_{k} \partial x_{k}} \\
T_{j}=\frac{\partial T}{h_{j} \partial x_{j}}, \quad T_{j k}=\frac{\partial T_{j}}{h_{k} \partial x_{k}} \\
\rho_{j}=\frac{\partial \rho}{h_{j} \partial x_{j}}, \quad \rho_{j k}=\frac{\partial \rho_{j}}{h_{k} \partial x_{k}}
\end{gathered}
$$

The omitted terms in ( ..) of Eqs. (13) and (14) can be obtained from the corresponding complete expression in Eq. (12) by index permutations, and the $\alpha_{l}$ can be found in Ref. 16 .

Similarly,

$$
\begin{aligned}
& \sigma_{12}^{(2)}=\sigma_{21}^{(2)}={ }_{P}^{\prime 2}\left(\beta_{1} u_{11} u_{12}+\beta_{2} u_{12} u_{22}+\beta_{2} u_{11} u_{21}+\beta_{1} u_{21} u_{22}\right. \\
& +\beta_{3} R\left(T_{12}+T_{21}\right)+\beta_{4} \frac{R T}{\rho}\left(\rho_{12}+\rho_{21}\right)+\beta_{5} \frac{R}{T} T_{1} T_{2} \\
& +\beta_{6} \frac{R T}{\rho^{2}} \rho_{1} \rho_{2}+\beta_{17} u_{13} u_{23}+\beta_{7} \frac{R}{\rho}\left(T_{2} \rho_{1}+T_{1} \rho_{2}\right) \\
& \left.+\beta_{8}\left(u_{12} u_{33}+u_{21} u_{33}\right)+\beta_{9}\left(u_{31} u_{23}+u_{31} u_{32}+u_{13} u_{32}\right)\right) \\
& +\frac{\mu^{2}}{p}\left(\beta_{10} R \frac{T_{2}}{r}+\beta_{11} \frac{R T}{\rho r} \rho_{2}+\beta_{2} \frac{u_{1}}{r} u_{12}+\beta_{1} \frac{u_{1}}{r} u_{21}\right. \\
& \left.+\beta_{13} \frac{u_{2}}{r} u_{11}+\beta_{14} \frac{u_{2}}{r} u_{22}+\beta_{15} \frac{u_{2}}{r} u_{33}+\beta_{14} \frac{u_{2} u_{1}}{r^{2}}\right) \\
& \left.\quad+\beta_{12} \frac{u_{2}}{r} u_{13}+\beta_{12} \frac{u_{2}}{r} u_{31}\right) \\
& \left.+\beta_{12} \frac{u_{2}}{r} u_{23}+\beta_{16} \frac{u_{2}}{r} u_{32}\right) \\
& \sigma_{23}^{(2)}=\sigma_{32}^{(2)}=\frac{\mu^{2}}{p}(\cdots)+\frac{\mu^{2}}{p}\left(\beta_{1} \frac{u_{1}}{r} u_{23}+\beta_{2} \frac{u_{1}}{r} u_{32}\right. \\
& +\beta_{8} \frac{\mu_{1}}{r} u_{13}+\beta_{8} \frac{u_{1}}{r} u_{31}
\end{aligned}
$$

where the omitted terms in Eqs. (16) and (17) can be obtained from the corresponding complete expression in Eq. (15)by index permutations, and the $\beta_{i}$ can be found in Ref. 16.

For Bumett heat-flux terms,

$$
\begin{aligned}
& q_{1}^{(2)}=\frac{\mu^{2}}{\rho}\left(\gamma_{1} \frac{1}{T} T_{1} u_{11}+\gamma_{2} \frac{1}{T} T_{1} u_{22}+\gamma_{3} u_{111}+\gamma_{5} u_{221}\right. \\
& +\gamma_{4} u_{212}+\gamma_{6} \frac{1}{T} T_{2} u_{21}+\gamma_{7} \frac{1}{T} T_{2} u_{12}+\gamma_{8} \frac{1}{\rho} \rho_{1} u_{11} \\
& +\gamma_{9} \frac{1}{\rho} \rho_{1} u_{22}+\gamma_{10} \frac{1}{\rho} \rho_{2} u_{12}+\gamma_{10} \frac{1}{\rho} \rho_{2} u_{21}+\gamma_{2} \frac{1}{T} T_{1} u_{33} \\
& +\gamma_{5} u_{331}+\gamma_{9} \frac{1}{\rho} \rho_{1} u_{33}+\gamma_{6} \frac{1}{T} T_{3} u_{31}+\gamma_{10} \frac{1}{\rho} \rho_{3} u_{13} \\
& \left.+\gamma_{10} \frac{1}{\rho} \rho_{3} u_{31}+\gamma_{7} \frac{1}{T} T_{3} u_{13}+\gamma_{4} u_{122}+\gamma_{4} u_{133}+\gamma_{4} u_{313}\right) \\
& +\frac{\mu^{2}}{\rho}\left(\gamma_{13} \frac{1}{T r} u_{2} T_{2}+\gamma_{2} \frac{1}{T r} u_{1} T_{1}+\gamma_{3} \frac{u_{11}}{r}+\gamma_{14} \frac{u_{1}}{r^{2}}\right. \\
& \left.+\gamma_{9} \frac{1}{\rho r} u_{1} \rho_{1}+\gamma_{15} \frac{1}{\rho r} u_{2} \rho_{2}+\gamma_{16} \frac{u_{22}}{r}\right)
\end{aligned}
$$

$$
\begin{aligned}
q_{2}^{(2)} & =\frac{\mu^{2}}{\rho}(\cdots)+\frac{\mu^{2}}{\rho}\left(\gamma_{1} \frac{1}{T r} u_{1} T_{2}+\gamma_{17} \frac{u_{12}}{r}+\gamma_{18} \frac{1}{T r} u_{2} T_{1}\right. \\
& \left.+\gamma_{15} \frac{1}{\rho r} u_{2} \rho_{1}+\gamma_{8} \frac{1}{\rho r} u_{1} \rho_{2}+\gamma_{4} \frac{u_{21}}{r}+\gamma_{19} \frac{u_{2}}{r^{2}}\right) \\
q_{3}^{(2)} & =\frac{\mu^{2}}{\rho}(\cdots)+\frac{\mu^{2}}{\rho}\left(\gamma_{2} \frac{1}{T r} u_{1} T_{3}+\gamma_{12} \frac{u_{13}}{r}\right. \\
& \left.+\gamma_{9} \frac{1}{\rho r} u_{1} \rho_{3}+\gamma_{4} \frac{u_{31}}{r}\right)
\end{aligned}
$$




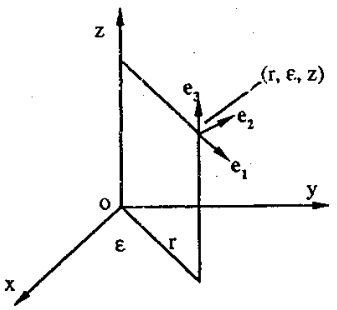

Fig. 2 Axisymmetric coordinates.

where the omitted terms in Eqs. (19) and (20) can be obtained from the corresponding complete expression in Eq. (18) by index permutations, and the $\gamma_{i}$ can be found in Ref. 16.

\section{Numerical Methods}

The finite-volume formulation of the conservation equations for an axisymmetric grid cell as in Fig. 2 is

$$
\begin{aligned}
& \frac{\partial \tilde{U} V_{i j}}{\partial t}+\left(S_{i+\frac{1}{2}} \boldsymbol{F}_{i+\frac{1}{2}}^{\prime}-S_{i-\frac{1}{2}} \boldsymbol{F}_{i-\frac{1}{2}}^{\prime}\right) \\
& \quad+\left(S_{j+\frac{1}{2}} \boldsymbol{F}_{j+\frac{1}{2}}^{\prime}-S_{j-\frac{1}{2}} \boldsymbol{F}_{j-\frac{1}{2}}^{\prime}\right)-\boldsymbol{H}_{i j} S_{i j}=0
\end{aligned}
$$

where

$$
\begin{gathered}
\left.\boldsymbol{U}=\mid \begin{array}{c}
\rho \\
\rho u \\
\rho v \\
e
\end{array}\right\}, \quad \boldsymbol{H}=\left\{\begin{array}{c}
0 \\
0 \\
p+\sigma_{\epsilon} \\
0
\end{array} \mid\right. \\
\boldsymbol{F}^{\prime}=(\boldsymbol{F i}+\boldsymbol{G} \boldsymbol{j}) \cdot n \\
\left.\boldsymbol{F}=\mid \begin{array}{c}
\rho u \\
\rho u^{2}+p+\sigma_{x} \\
\rho u v+\sigma_{x y} \\
\left(e+p+\sigma_{x}\right) u+\sigma_{x y} v+q_{x}
\end{array}\right\} \\
\boldsymbol{G}=\left|\begin{array}{c}
\rho v \\
\rho u v+\sigma_{y x} \\
\rho v^{2}+p+\sigma_{y} \\
\left(e+p+\sigma_{y}\right) v+\sigma_{y x} u+q_{y}
\end{array}\right|
\end{gathered}
$$

The expressions of Burnett stress and heat-flux terms for axisymmetric flow can be obtained as a special case of Eqs. (12-20) by assuming

$$
\begin{gathered}
x_{1}=y . \quad x_{2}=\epsilon, \quad x_{3}=x \\
u_{1}=u, \quad u_{2}=0, \quad u_{3}=u, \quad \partial / \partial \epsilon=0
\end{gathered}
$$

Equation (21) is solved by a second-order accurate implicit numerical method, which is an extension of the implicit method for solving the planar two-dimensional augmented Burnett equations!

\section{Boundary Conditions and Uniqueness of Solutions}

As discussed in the introduction, we only study hypersonic flowfields near the stagnation point on a cold wall at low Knudsen numbers. For such flowfields, the Burnett equations are different from the Navier-Stokes equations mainly in the bow shock, which is away from the wall. Therefore, the first-order slip conditions' are used in the numerical method for both the Navier-Stokes and the Burnett equations:

$$
\begin{gathered}
u_{s}=\frac{2-{ }_{\mathrm{A}} 0 \mathrm{au}}{\sigma}+\frac{3}{4} \frac{\mu}{\rho T} \frac{\partial T}{a x} \\
T_{s}=T_{\text {wall }}+\frac{2-\alpha}{\alpha} \frac{2 \gamma}{(\gamma+1) \operatorname{Pr}} \lambda \frac{\partial T}{\partial y}
\end{gathered}
$$

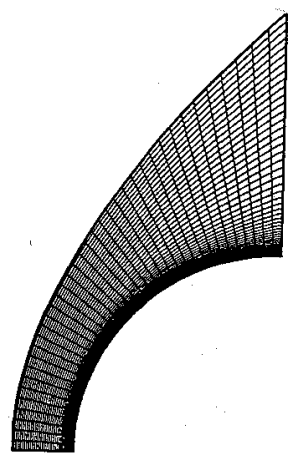

Fig. 3 Typical $76 \times 120$ grid used in the computations.

Since no additional boundary conditions are available, the highorder terms in the augmented Burnett equations are computed by an extrapolation method from the variables in the interior of the flowfields. If the Burnett terms are significant on the surface, this numerical treatment may be questionable concerning the uniqueness of the solutions. Therefore, the present augmented Burnett solutions can only be applied to the cases where the Burnett terms are not significant on the wall. A unique set of solutions for the augmented Burnett equations can be obtained for such flowfields.

\section{Results and Discussions}

Numerical solutions of the augmented Burnett equations in the stagnation region of hypersonic flow over a sphere were obtained.

Hypersonic flow over a sphere can be characterized by $\mathrm{Kn}, \quad M_{\infty}$, and $T_{\text {wall }} / T_{\infty}$. For high $M_{\infty}$ and low $K_{n}$, translational nonequilibrium of the flow mainly occurs within the shock layer, whose thickness depends on $M_{\infty}$ and $K n$, and occurs on the wall surface to a less extent. When $K n$, $\ll 1$, the Navier-Stokes equations are valid throughout the flowfield in the stagnation region except in a negligiblely thin area across the bow shock, which can be treated as a mathematical discontinuity surface. As $K n$, increases, however, the thickness of the shock wave and importance of resolving the structure of the nonequilibrium shock layer also increase. The augmented Burnett solutions were compared with the NavierStokes and the DSMC solutions for such flowfields.

An implicit computer code was written for solving the axisymmetric Navier-Stokes and augmented Burnett equations by modifying our previous planar two-dimensional code for the Burnett equations. The numerical accuracy of the results presented in this paper has been checked by grid refinement studies discussed in detail in Ref. 16. The present Navier-Stokes solutions were also tested by comparing them with experimental results for hypersonic flow in the continuum regime. ${ }^{16}$ The computer code, which has not been optimized for vectorized computations on a Cray-90 computer, takes about $13 \mathbf{s}$ for the Navier-Stokes and $20 \mathbf{s}$ for the augmented Burnett calculations of an iteration using $54 \times 86$ grids. Figure 3 shows a typical grid used in the computations. It typically takes about 1000 iterations to reach steady-state solutions. Code optimization is needed if the code is to be used extensively.

Case 1: Monatomic Gas $\left(M_{\infty}=10.95, K n, \quad=0.2\right)$

The flow conditions of this case were $M_{\infty}=10.95, K n,=0.2$, $T_{\text {wall }}=\mathrm{T},=300 \mathrm{~K}, P r=2 / 3, \mathrm{y}=5 / 3$, and $\bar{r}=0.02 \mathrm{~m}$. The wall was a diffuse-reflection wall, and the gas model was the hardsphere model, whose viscosity coefficient is $\mu=\mu_{0}\left(T / T_{0}\right)^{0.5}$. A set of $102 \times 168$ grids was used in the computations.

Because DSMC results were not available for this case, we only compared the augmented Burnett solutions with the corresponding Navier-Stokes solutions. The temperature and density contours are shown in Figs. 4 and 5, respectively. The temperature, velocity, and density distributions along the stagnation streamline are shown in Figs. 6-8. These results show that the main differences between the augmented Burnett and Navier-Stokes solutions are in the temperature profile. The augmented Burnett solutions predict a thicker bow shock than the Navier-Stokes equations, whereas the Navier-Stokes equations have been found to predict thinner shocks than those of 


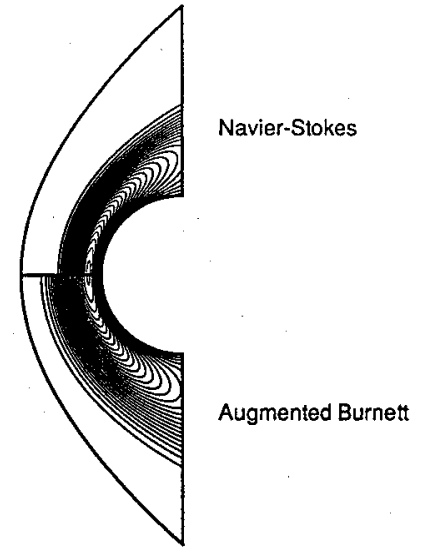

Fig. 4 Flowfield temperature contours $\left(M_{\infty}=10.95, K n_{\infty}=0.2\right)$.

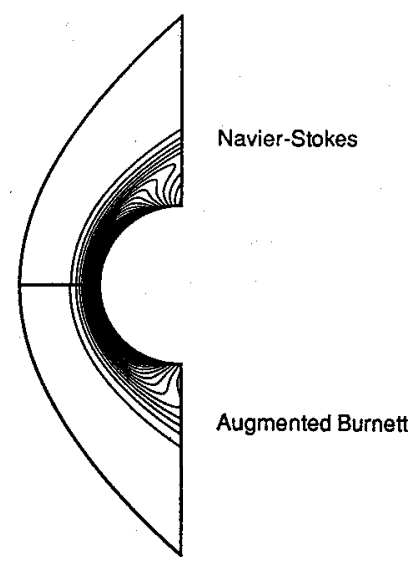

Fig. 5 Flowfield density contours $\left(M_{\infty}=10.95, K n_{\infty}=0.2\right)$.

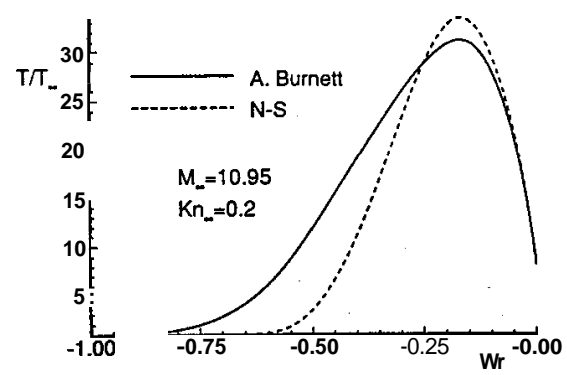

Fig. 6 Temperaturedistribution along stagnation streamline.

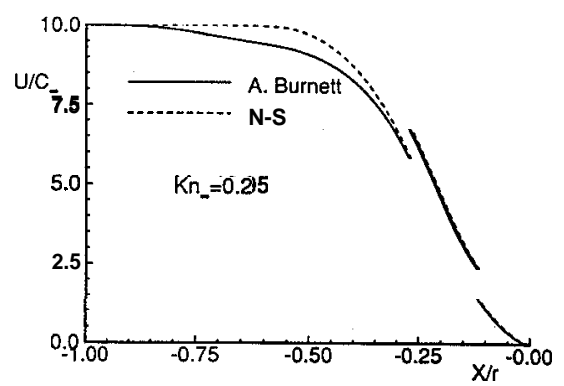

Fig. 7 Velocity distribution along stagnation streamline.

experimental and DSMC results. The maximum temperature along the stagnation line for the augmented Bumett equations is lower than that of the Navier-Stokesequations. However, the density distribution does not show much difference across the shock.

We next examined the departure of local transnational nonequilibrium measured by $\left|\sigma_{x} / p\right|$ for this case of Knudsen number 0.2 , which was quite large for a continuum approach. Figures 9-11 show the profiles of $\sigma_{x} / p, p / p_{\infty}$, and $\boldsymbol{q}_{x} / \mathbf{1} / 2\left(\rho_{\infty} c_{\infty}^{3}\right)$ across the stagnation

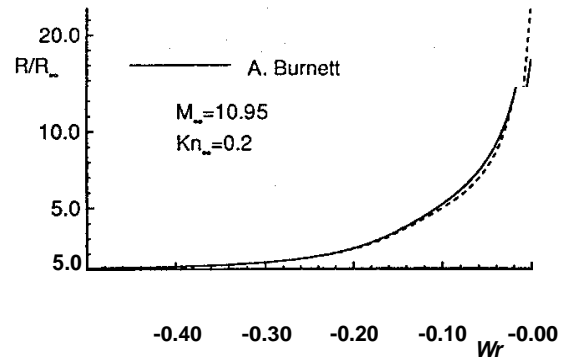

Fig. 8 Density distribution along stagnation streamline.

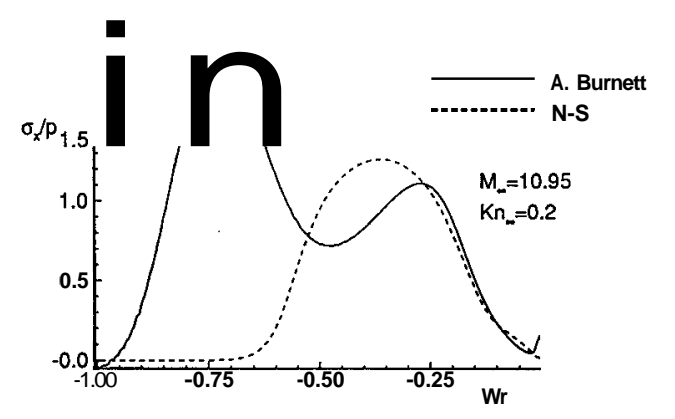

Fig. 9 Ratio of viscous stress to thermodynamic pressure along stagnation streamline.

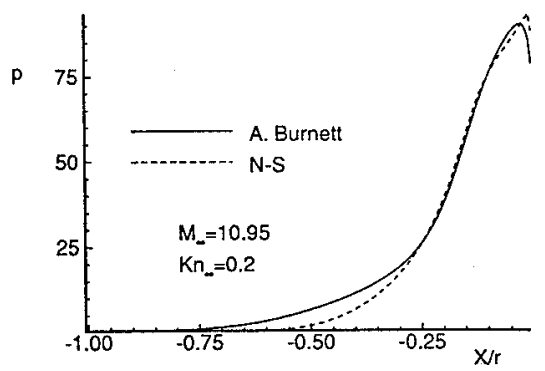

Fig. 10 Pressure distribution along stagnation streamline.

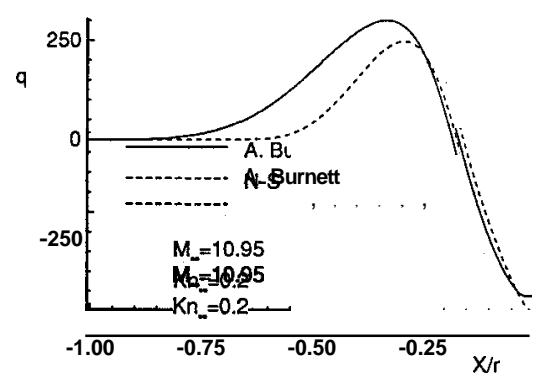

Fig. 11 Heat-flux distributionalong stagnation streamline.

line. The figures show that the main differences between the NavierStokes and the augmented Bumett equations occur inside the shock layer. The value of $\sigma_{x} / p$ is very large in the upstream portion of the shock structure $(x / \bar{r}<-0.5)$, which means that the flow is in strong translational nonequilibrium there. Under such conditions, both the augmented Bumett and the Navier-Stokesequations are inaccurate. The question here is whether the augmented Bumett equations are more accurate in predicting the failure of the Navier-Stokes equations. It will be interesting if the corresponding profile of DSMC results is also available for comparison.

It was also noted that the Navier-Stokes and augmented Bumett solutions have some differences near the surface. However, the differences seem to have little effect on the overall solutions because the value of $\sigma_{x} / p$ is much smaller on the wall. However, if $\mathbf{K n}$, is larger, the uniqueness of the augmented Bumett solutions may become questionable because of the uncertainty in boundary conditions used in the numerical solutions. 
To summarize the results of this case, the flow is in strong translational nonequilibrium across the shock layer. The augmented Burnett equations predict a different flowfield from the Navier-Stokes equations, mainly in the temperature profiles. The augmented Burnett equations also predict a thicker bow shock than the NavierStokes equations based on temperature profiles. This property of the augmented Burnett solutions may be significant for studying the flowfields sensitive to the temperature profiles, if the augmented Burnett solutions are indeed proven to be more adequate. If this is the case, then, because of the uncertainty in the boundary conditions the practical applications of the Burnett equations may be limited to the flowfields inside the shock layer, that is, away from the wall surface.

\section{Case 2 Comparison with DSMC and VSL Results}

We compared the augmented Burnett solutions with the NavierStokes solutions, a viscous shock layer (VSL) solution, ${ }^{17}$ and DSMC results. ${ }^{18}$ The flow conditions are $M_{\infty}=10, K n,=0.1$, $T_{\text {wall }} / T_{\infty}=1.0$, and the gas model was the same as the one in case 1 .The numerical solutions were obtained using $54 \times 86$ grids.

Density and temperature profiles along the stagnation line are plotted in Figs. 12 and 13, respectively. The augmented Burnett results show closer agreement with DSMC results than the NavierStokes results, the VSL Navier-Stokes results, ${ }^{17}$ and the shocklayer results" do. From the temperature profile, we can see that the augmented Burnett equations predict a thicker shock layer than the Navier-Stokes equations, but a thinner layer than the one predicted by the DSMC method. The density profiles for both the NavierStokes and the augmented Burnett equations show little difference from the DSMC results.

However, there is a significant discrepancy between our NavierStokes solutions and the VSL solutions, ${ }^{17}$ which are also solutions of the Navier-Stokes equations. Our numerical tests show that the discrepancy is not caused by different viscosity-coefficient models used. In order to further validate our Navier-Stokes code, we compared our Navier-Stokes solutions with the VSL solutions under conditions that the Aeroassist Flight Experiment (AFE) vehicle encounters at times $t=10$ to $30 \mathrm{~s}$ after its re-entry into the atmosphere. ${ }^{20}$ Reference 18 computed the flowfields using a VSL and a hypersonic merged-layer formulation of the Navier-Stokes equations (HMLO). The flow conditions of the three cases are given in Table 1 . The viscosity coefficient was computed by Sutherland's formula for air with $\operatorname{Pr}=0.72$ and $\gamma=1.4$. Figures 14-16 show the stagnation-linetemperature for the three runs. Our NavierStokes results are in good agreement with those of Ref. 18,especially in the cases of lower $\mathrm{Kn}$. Therefore, the current Navier-Stokes

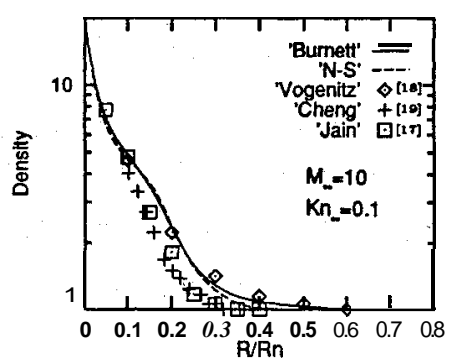

Fig. 12 Density distribution along stagnation streamline.

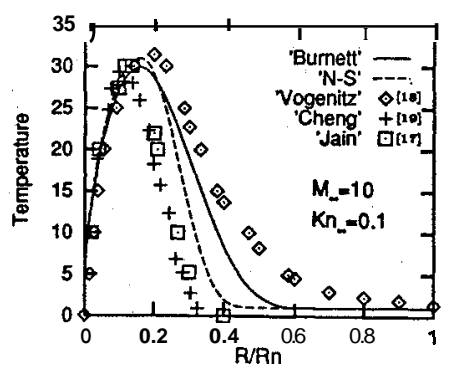

Fig. 13 Temperature distribution along stagnation streamline.
Table 1 Flow conditions of the three runs in case 2

\begin{tabular}{cccllcc}
\hline \hline $\boldsymbol{t ( s )}$ & $M_{\infty}$ & $T_{\infty}, \mathbf{K}$ & $\boldsymbol{R e}$, & $\mathrm{Kn}$, & $\overline{\boldsymbol{r}}, \mathbf{m}$ & $T_{\text {wall }}, \mathrm{K}$ \\
\hline 15 & 31.19 & 251.2 & 151.0 & 0.312 & 2.53 & 1111 \\
20 & 32.54 & 230.8 & 278.0 & 0.1768 & 2.53 & 1111 \\
30 & 34.66 & 203.7 & 986.9 & 0.059 & 2.53 & 1111 \\
\hline \hline
\end{tabular}

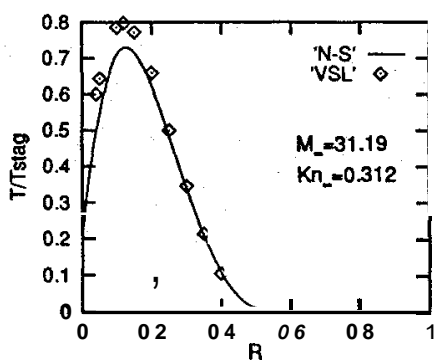

Fig. 14 Temperature distribution along stagnationstreamline.

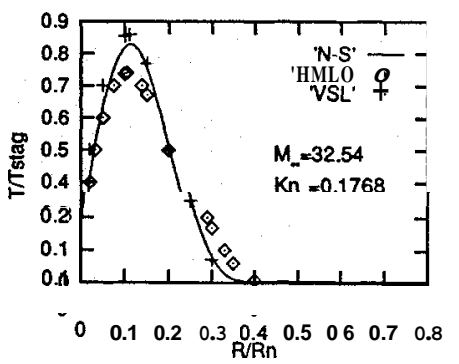

Fig. 15 Temperature distributionalong stagnation streamline.

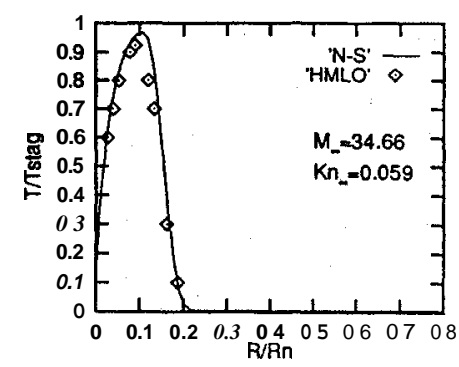

Fig. 16 Temperature distribution along stagnation streamline.

code can be considered reliable, and the discrepancies among the Navier-Stokes solutions shown in Fig. 13 may arise from possible differences in freestream conditions used.

With regard to the past observation ${ }^{\mathrm{g}}$ on the usefulness of the Burnett equations, the current results show that the augmented Burnett solutions are not very different from the Navier-Stokes solutions. Though both the Navier-Stokes and the augmented Burnett solutions are inaccurate inside the shock wave, the augmented Burnett solutions do seem to agree better with DSMC results than the Navier-Stokes solutions do.

\section{Case 3: Effect of $K n$, and M,}

We did two parametric studies on the effects of $K n$, and $M_{\infty}$. The flow conditions were those of the $t=20 \mathrm{~s}$ case in Table 1 .

Figures 17-20show typical results of the Knudsen-number study. A Mach number of 10 was chosen, and various values of $K n$ were used. The results for the cases of $K \boldsymbol{n}=0.05$ and 0.1768 are presented here. In general, the augmented Burnett equations predict thicker shock layers, based on the temperature distribution, than the Navier-Stokes equations do. The difference between the two becomes more pronounced at higher values of $\boldsymbol{K n}$. But the density profiles show little difference between the two.

Figures 17, 19, 21, and 22 show the effects of varying only Mach numbers at a fixed Knudsen number of 0.1768. Again, the augmented Burnett equations predict thicker shock layers and lower maximum temperatures than the Navier-Stokes equations do. 


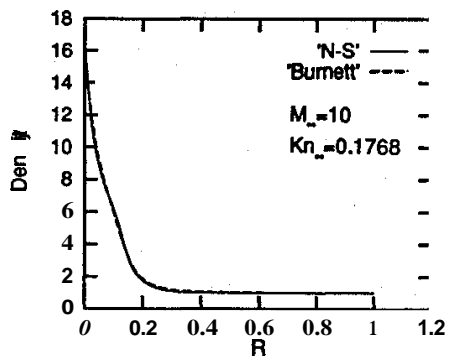

Fig. 17 Density distributionalong stagnation streamline.

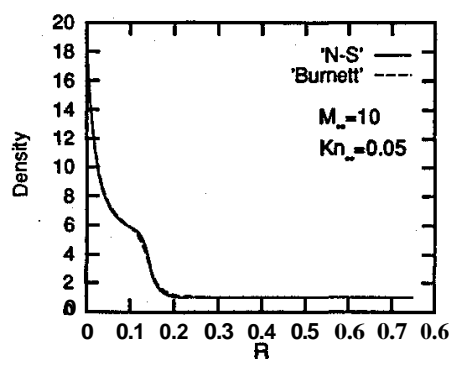

Fig. 18 Density distributionalong stagnationstreamline.

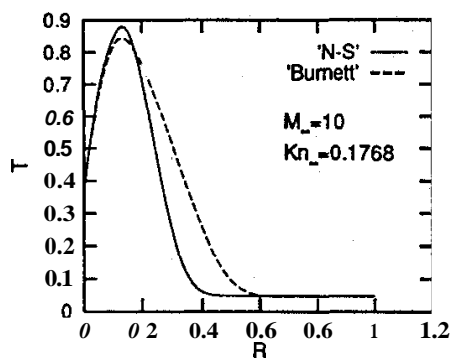

Fig. 19 Temperature distributionalong stagnation streamline.

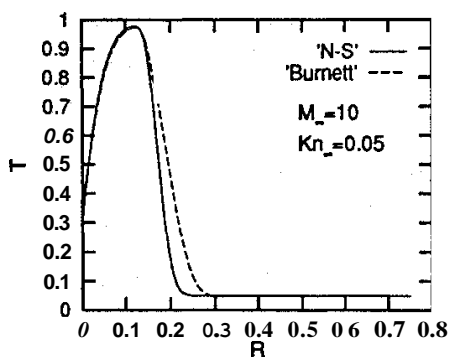

Fig. 20 Temperature distribution along stagnation streamline.

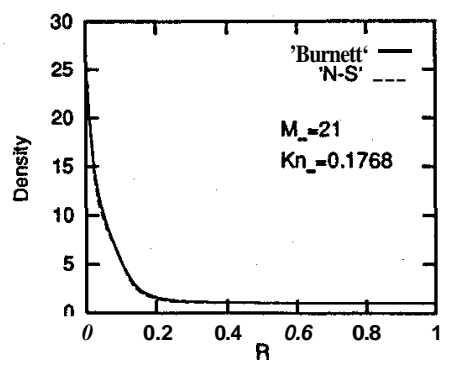

Fig. 21 Density distributionalong stagnationstreamline.

The normalized peak temperature decreases as the Mach number increases. However, the variation of the profiles as the Mach number increases is much less than the variation that occurs when $K \boldsymbol{n}$ is varied. Our calculations for the augmented Burnett equations in cases of $M_{\infty}$ higher than 25 lead to negative temperatures in the upstream portion of the bow shock. This difficulty, which may be caused by the instability of the Bumett equations, limits the range of Mach numbers in computing the Burnett solutions.

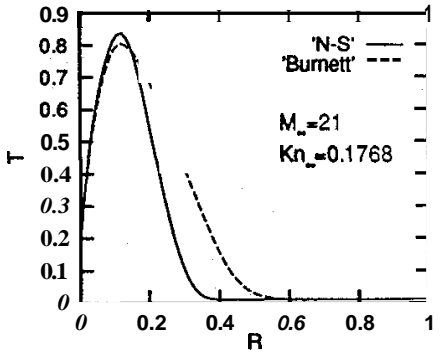

Fig. 22 Temperature distribution along stagnation streamline.

The results of this case show that the augmented Bumett solutions are not very different from the Navier-Stokes solutions except in the temperature profiles. Further studies are needed on the validity and significance of the augmented Bumett solutions for this flowfield.

\section{Summary and Conclusions}

The general three-dimensional components of the augmented Bumett stress and heat-flux terms have been derived from their tensor forms. Subsequently, augmented Bumett-equation solutions have been obtained for axisymmetric hypersonic flow over a sphere near the stagnation point. However, these solutions are restricted to the flow in the bow shock because of the uncertainty in the boundary conditions of the augmented Bumett equations.

The augmented Bumett solutions were studied through comparison with Navier-Stokes solutions and available DSMC results. The results partly confirmed the past observation that the Burnett equations make only insignificant improvement over the Navier-Stokes equations when the Navier-Stokes equations are adequate. An exception to that observation is that the augmented Burnett equations predict thicker shock layers than the Navier-Stokesequations based on temperature profiles. Though both the Navier-Stokes and the augmented Bumett equations are inaccurate in a strong shock, the augmented Bumett solutions seem to agree better, to some extent, with the DSMC results than with the Navier-Stokes equations. If the augmented Bumett solutions are indeed proven to be adequate, this property may be significant for studying flow where accurate prediction of the temperature profiles is important.

\section{Acknowledgments}

This research was supported by Air Force Office of Scientific Research Grant F49620-94-1-0019, with L. Sakell as the grant monitor. Cray C 90 computer time was provided by the DoD High Performance Computing Center. The authors would like to acknowledge helpful comments by the reviewers and the editors.

\section{References}

${ }^{1}$ Fiscko, K. A., "Study of ContinuumHigher OrderClosure Models Evaluated by a Statistical Theory of Shock Structure," Ph.D. Thesis, Stanford Univ., Stanford,CA, Aug. 1988.

${ }^{2}$ Fiscko, K. A., and Chapman, D. R., "Comparison of Burnett, SupertBumett, and Monte Carlo Solutions for Hypersonic Shock Structure," Rarefied Gas Dynamics: Theoretical and Computational Techniques, edited by E. P. Muntz, D. P. Weaver, and D. H. Campbell, Vol. 118, Progress in Astronautics and Aeronautics, AIAA, New York, 1988, pp. 374-395.

${ }^{3}$ Lumpkin, F. E., III, "Development and Evaluation of ContinuumModels for Translational-RotationalNonequilibrium,"Ph.D. Thesis, Stanford Univ., Stanford,CA, March 1990.

${ }^{4}$ Zhong, X., MacCormack, R. W., and Chapman, D. R., "Stabilization of the BumettEquations and Applicationto High-AltitudeHypersonicFlows," AIAA Journal, Vol. 31, No. 6,1993, pp. 1036-1043(AIAAPaper 91-0770).

${ }^{5}$ Zhong, X., MacCormack,R. W., and ChapmanD. R., "Evaluationof Slip Boundary Conditionsfor the BurnettEquations with Application to Hypersonic Leading Edge Flow," Proceeding of 4th International Symposium on CFD, Davis, CA, Vol. 2, 1991,pp. 1360-1366.

${ }^{6}$ Imlay, S. T., "Solution of the Bumett Equations for HypersonicFlows Near the ContinuumLimit," AIAA Paper 92-2922, June 1992.

${ }^{7}$ Liaw, G. S., Guo, K. L., and Chou,L. C., "Burnett Solutions Along the Stagnation Line of a Cooled Cylinder in Low-Density Hypersonic Flows," AIAA Paper 93-2726,July 1993. 
${ }^{8}$ Zhong, X., "On Numerical Solutions of Burnett Equations for Hypersonic Flow past 2-D Circular Blunt Leading Edges in ContinuumTransition Regime," AIAA Paper 93-3092, July 1993.

${ }^{9}$ Schaaf, S. A., and Chambre, P. L., Flow of Rarejied Gases, Princeton Aeronautical Paperbacks, Vol. 8, Princeton Univ. Press, Princeton, NJ, 1961.

"'Comeaux, K. A., Chapman, D. R., and MacCormack, R. W., "An Analysis of the Burnett Equations Based on the Second Law of Thermodynamics," AIAA Paper95-0415, Jan. 1995.

${ }^{11}$ Bobylev, A. V., "The Chapman-Enskogand Grad Methods for Solving the Boltzmann Equation,"Soviet Physics-Doklady, Vol. 27, No. 1, 1982, pp. 29-31.

${ }^{12}$ Pham-Van-Diep, G. C., Erwin, D. A., and Muntz, E. P., "Testing Continuum Descriptions of Low-Mach-NumberShock Structures," Journal of Fluid Mechanics, Vol. 232, 1991,pp. 403-413.

${ }^{13}$ Lee, C. J., "Unique Determination of Solutions to the Burnett Equations," AIAA Journal, Vol. 32, No. 5, 1994,pp. 985-990.

${ }^{14}$ Chapman, S., and Cowling, T. G., The Mathematical Theory of NonUniform Gases, 3rd ed., Cambridge Univ. Press, 1970.

${ }^{15}$ Wang Chang, C. S., and Uhlenbeck, G. E., "On the Transport
Phenomena in Rarefied Gases," Studies in Statistical Mechanics, Vol. 5, 1948,pp. 1-17.

${ }^{16}$ Zhong X., andFurumoto, G. H., "Solutionsof the BurnettEquationsfor Axisymmetric HypersonicFlow past SphericalBlunt Bodies," AIAA Paper 94-1959, June 1994

${ }^{17}$ Jain,A. C., "HyprsonicMerged LayerFlow over'a Sphere," AIAAPaper 85-1031, 1985.

${ }^{18}$ Vogenitz, F. W., and Takata, G. Y., "Monte Carlo Study of Blunt Body Hypersonic Viscous Shock Layers," Rarejied Gas Dynamics, edited by D. Dini, C. Cercignani, and S. Nocilla, Editrice Tecnico Scientifica, Pisa, Italy, 1971,pp. 519-528.

${ }^{19}$ Cheng, H. K., "Viscous Hypersonic Blunt-Body Problems and the Newtonian Theory," Fundamental Phenomena in Hypersonic Flow, edited by J. G. Hall, Cornell Univ. Press, 1966,pp. 90-131.

${ }^{20}$ Jain, A. C., and Woods, H. G., "Investigation of Hypersonic Rarefied Flow on a SphericalNose of the AOTV," Final Report, NASA-CR-179153, 1987.

I. D. Boyd Associate Editor 